\title{
Pseudo-planar Ge-on-Si single photon avalanche diode detector with record low noise-equivalent power
}

Millar, Ross, Kirdoda, Jaroslaw, Thorburn, Fiona, Yi, Xin, Greener, Zoë, et al.

Ross W. Millar, Jaroslaw Kirdoda, Fiona Thorburn, Xin Yi, Zoë M. Greener, Laura Huddleston, Bhavana Benakaprasad, Scott Watson, Conor Coughlan, Gerald S. Buller, Douglas J. Paul, "Pseudo-planar Ge-on-Si single photon avalanche diode detector with record low noise-equivalent power," Proc. SPIE 11881, Quantum Technology: Driving Commercialisation of an Enabling Science II, 118810F (6 October 2021); doi: 10.1117/12.2599033

SPIE. Event: SPIE Photonex, 2021, Glasgow, Scotland, United Kingdom 


\title{
Pseudo-planar Ge-on-Si Single-Photon Avalanche Diode Detector with Record Low Noise-Equivalent Power
}

\author{
Ross W. Millar ${ }^{1}$, Jaroslaw Kirdoda ${ }^{1}$, Fiona Thorburn², Xin Yi², Zoë M. Greener ${ }^{2}$, \\ Laura Huddleston ${ }^{2}$, Bhavana Benakaprasad ${ }^{1}$, Scott Watson ${ }^{1}$, Conor Coughlan ${ }^{1}$, Gerald S. Buller ${ }^{2}$ and \\ Douglas J. Paul ${ }^{1}$ \\ ${ }^{1}$ University of Glasgow, James Watt School of Engineering, Rankine Building, \\ Glasgow, G12 8LT, U.K. \\ ${ }^{2}$ Institute of Photonics and Quantum Sciences, School of Engineering and Physical Sciences, Heriot- \\ Watt University, Edinburgh EH14 4AS, UK
}

\begin{abstract}
Single-photon avalanche diode (SPAD) detectors are of significant interest for numerous applications, including light detection and ranging (LIDAR), and quantum technologies such as quantum-key distribution and quantum information processing. Here we present a record low noise-equivalent-power (NEP) for Ge-on-Si SPADs using a pseudo-planar design, showing high detection efficiency in the short-wave infrared; a spectral region which is key for quantum technologies and hugely beneficial for LIDAR. These devices can leverage the benefits of Si avalanche layers, with lower afterpulsing compared to InGaAs/InP, and reduced cost due to Si foundry compatibility. By scaling the SPAD pixels down to $26 \mu \mathrm{m}$ diameter, a step change in performance has been demonstrated, with significantly reduced dark count rates (DCRs), and low jitter (134ps). Ge-on-Si SPADs were fabricated using photolithography techniques and characterised using time-correlated single-photon counting. The DCR reaches as low as kilocount/s at $100 \mathrm{~K}$ for excess bias up to $\sim 5 \%$. This reduction in DCR enables higher temperature operation; e.g. the DCR of a $26 \mu \mathrm{m}$ diameter pixel at $150 \mathrm{~K}$ is approximately equivalent to a $100 \mu \mathrm{m}$ diameter pixel at $77 \mathrm{~K}(100 \mathrm{~s}$ of kilocounts/s). These low values of DCR, coupled with the relatively temperature independent single photon detection efficiencies (SPDE) of $\sim 29 \%$ (at $1310 \mathrm{~nm}$ wavelength) leads to a record low NEP of $7.7 \times 10^{-17} \mathrm{WHz}^{-1 / 2}$. This is approximately 2 orders of magnitude lower than previous similarly sized mesa-geometry Ge-on-Si SPADs. This technology can potentially offer a lowcost, Si foundry compatible SPAD operating at short-wave infrared wavelengths, with potential applications in quantum technologies and autonomous vehicle LIDAR.
\end{abstract}

Keywords: Single photon detector, quantum, lidar, SPAD, quantum technology, Germanium, Detector, Silicon photonics.

\section{INTRODUCTION}

Single-photon avalanche diode (SPAD) detectors are semiconductor devices capable of detecting the arrival of single photons with picosecond timing precision. Si based CMOS SPADs are becoming ubiquitous with billions of time-of-flight chips sold in smartphones globally for proximity detection, camera auto-focus assist and 3D LIDAR imaging. This CMOS technology, however, is limited to absorption at wavelengths in the near-infrared (NIR) below $1000 \mathrm{~nm}$, and is therefore best suited for short-range LIDAR applications where low laser powers can be used that are compliant with eye-safety regulations. Moving to the short-wave infrared (SWIR) offers numerous benefits for LIDAR systems [1]; significantly higher laser powers can be used whilst remaining eye-safe (IEC-60825-1 Standard), solar background radiation is lower than the NIR [2], and there is high atmospheric transmission [3]. The combination of these advantages means that LIDAR systems can operate at long-range in a variety of weather conditions, which are key needs of applications such as autonomous vehicle LIDAR and military range-finding [4,5]. Furthermore, operation at telecoms wavelengths in the SWIR enables SPADs to be used for a range of quantum communication and information processing applications [6], by detecting single photons sent in free-space or via optical fibre $[7,8]$. The current SPAD technology capable of SWIR operation is based on InGaAs/InP SPAD detectors which have expensive fabrication processes, and also suffer from effects such as high afterpulsing, which can severely limit the data acquisition rate of these devices [9]. Here we present results from technology based on the Ge-on-Si material platform, which can operate in the SWIR but using Si foundry compatible growth and processing [10-13], giving it the potential for low-cost at mass-volume production. We demonstrate that a

Quantum Technology: Driving Commercialisation of an Enabling Science II, edited by Miles J. Padgett, Kai Bongs, Alessandro Fedrizzi, Alberto Politi, Proc. of SPIE Vol. 11881, 118810F

(C) 2021 SPIE · CCC code: 0277-786X/21/\$21 - doi: 10.1117/12.2599033 
pseudo-planar device design can substantially improve the sensitivity of Ge-on-Si SPADs compared to simple mesa etched designs [14,15], yielding a step-change in performance, with $26 \mu \mathrm{m}$ diameter devices showing record low noise-equivalent powers [16].

\section{SPAD DESIGN}

Ge-on-Si SPADs use a separate absorption and multiplication design, whereby the photon is absorbed in the Ge layer, before the photo-generated electron drifts to the i-Si multiplication layer where it undergoes gain via impact ionization, producing a macroscopic current pulse that can be detected readily with external read-out electronics. A charge sheet layer is used to mediate the electric field between the two layers when the structure is under reverse bias, ensuring the $\mathrm{Si}$ avalanche region is above avalanche breakdown and a moderate electric-field is present in the Ge that is below breakdown but sufficient for electrons to drift into the Si. All previous Ge-on-Si SPADs used an in-situ doped charge sheet layer, combined with a mesa etch to define the pixel location [17,18]. Mesa designs can lead to high electric fields at the etched sidewall, where there is significant defect densities that act as generation-recombination centers, also increasing the likelihood of preferential edge breakdown due to electric-field hotspots. We have used a pseudo-planar design [14], as shown in Fig. 1, which uses an implanted charge sheet layer, and a local top contact to define the active area of the pixel away from etched sidewalls. Here, we scale the technology to a $26 \mu \mathrm{m}$ diameter charge sheet, with an optical access area of $13 \mu \mathrm{m}$ diamater, demonstrating significant improvement in noise-equivalent power (NEP) and jitter compared to all previous Ge-on-Si SPADs.

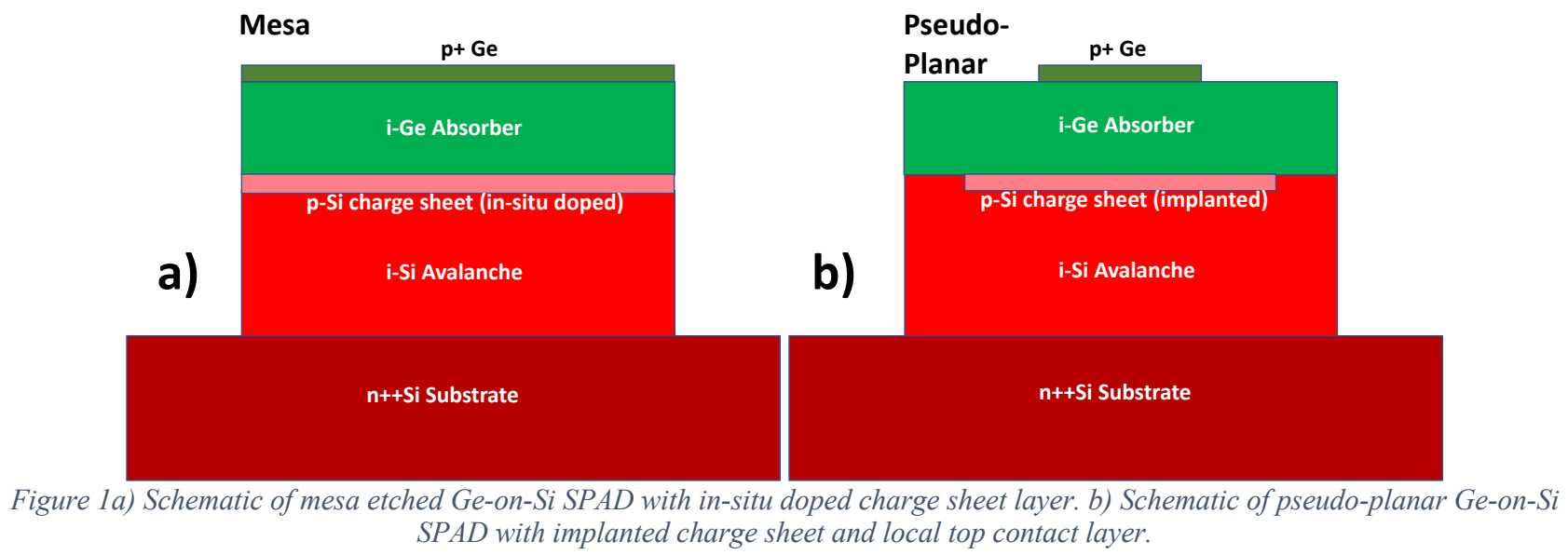

\section{WAFER GROWTH}

SPAD wafers are fabricated starting with $200 \mathrm{~mm}$ n+-Si doped wafers. An avalanche layer is grown at IQE Si with a thickness of $1.5 \mu \mathrm{m}$, ensuring a high triggering probability for injected electrons from the Ge absorber. Subsequently, wafers are patterned by photolithography to open windows in a photo-resist layer, to determine the position of charge sheet regions. Charge sheets are subsequently implanted using Boron, at an energy of $10 \mathrm{keV}$ and a dose of $3 \mathrm{e}^{2} 2 \mathrm{~cm}^{-2}$ before wafers are cleaned and the dopants are activated in a Rapid Thermal Anneal tool at $900^{\circ} \mathrm{C}$ for $30 \mathrm{~s}$. Subsequently, a $1 \mu \mathrm{m}$ $\mathrm{i}-\mathrm{Ge}$ absorber layer and $50 \mathrm{~nm}$ p-Ge top contact layer is grown at IQE using the double temperature growth technique [19] before the wafer is diced into $1 \mathrm{~cm}^{2}$ chips for processing. 


\section{SPAD FABRICATION}
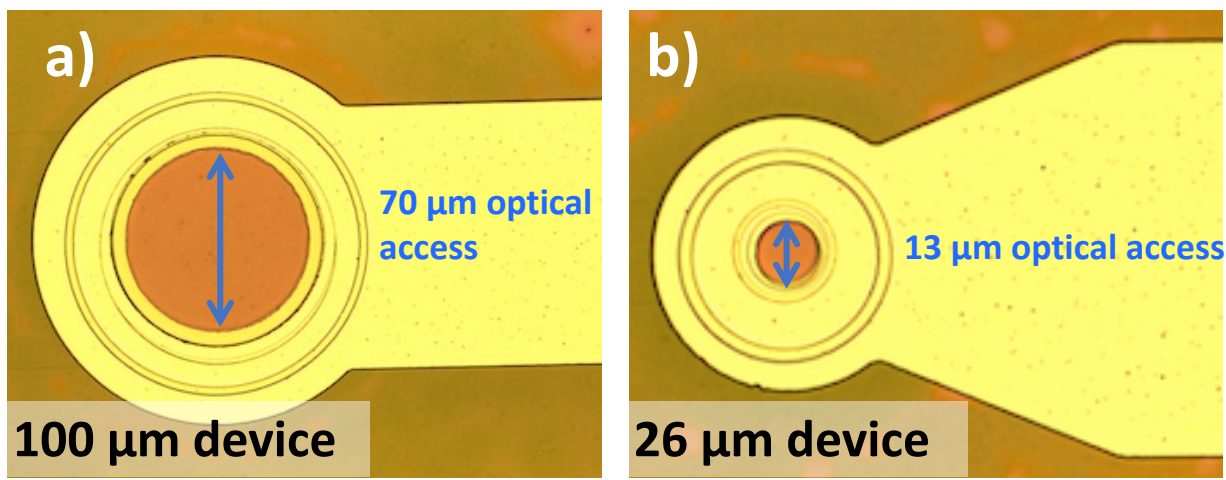

Figure 2: Optical microscope image of a) $100 \mu \mathrm{m}$ diameter charge sheet device and b) a $26 \mu$ m diameter charge sheet device. The images show the metal bond-pad region for wire bonding to a chip carrier. The diameters of the optical openings are shown.

As discussed previously, the devices require a local top contact layer to define the active area of the device in conjunction with the locally doped charge sheet. The top contact $\mathrm{p}+$ layer is patterned by photolithography and etched by $\sim 75 \mathrm{~nm}$ in an RIE tool using a fluorine-based chemistry [20] which has demonstrated low electrical damage in silicon devices with sub-10 nm widths [21]. Subsequently, a second 'buffer' etch is carried out to electrically isolate the devices laterally, at a distance of $10 \mu \mathrm{m}$ from the circumference of the implanted charge sheet. Devices are subsequently passivated with a $\mathrm{GeO}_{2}$ layer and capped by $\mathrm{Si}_{\mathrm{x}} \mathrm{N}_{\mathrm{y}}$ to prevent the water soluble oxide being damaged by the atmosphere [22]. The thickness of the $\mathrm{Si}_{\mathrm{x}} \mathrm{N}_{\mathrm{y}}$ layer is designed to act as an anti-reflection coating for the $1310 \mathrm{~nm}$ wavelength. The devices are subsequently planarized with a patternable oxide (hydrogen silsesquioxane) before via holes are defined, and metal contacts and bondpads are deposited with a metal evaporation tool. The $\mathrm{GeO}_{2}$ oxide passivation process has been previously demonstrated to have low frequency dispersion in Capacitance-Voltage measurements and shown to enhance photoluminescence intensity of Ge micro-crystals [23]. Figure 2 shows optical microscope images of the completed devices. The SPAD chips are subsequently wire-bonded to a chip-carrier to enable characterization in an optical cryostat.

\section{CHARACTERIZATION}

Ge-on-Si SPADs are characterized in a gated mode, where an AC voltage is superimposed on a fixed DC bias, to bias the devices above breakdown, making them sensitive to single photons. The characterization setup is shown in Fig 3a) and
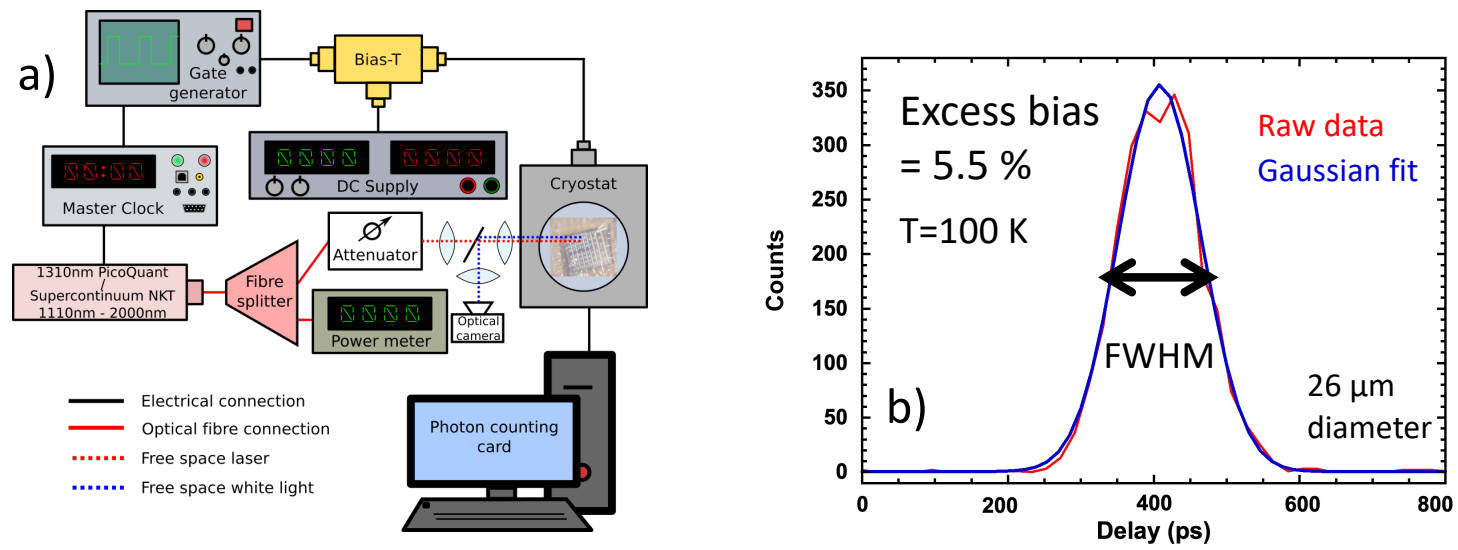

Figure 3a) A schematic diagram of the characterization setup. b) The timing histogram recorded by the photon counting card electronics of a $26 \mu \mathrm{m}$ diameter SPAD under illumination at $1310 \mathrm{~nm}$, operated at $100 \mathrm{~K}$ and an excess bias of $5.5 \%$. 
described in further detail in [14]. Devices are mounted in an optical cryostat and illuminated with a pulsed laser with a $<50 \mathrm{ps}$ pulse duration, where the laser pulse is incident in synchronization with the electrical gate. Attenuating the laser pulse to contain $<0.01$ photons per pulse ensures that there is a statistically negligible probability of a pulse containing $>$ 1 photon, which is key for achieving an accurate single photon detection efficiency (SPDE). The SPAD trigger events during the gate are logged using a time-correlated single-photon counting card, and a histogram is generated with and without optical illumination in order to determine the single photon detection efficiency (SPDE) and the dark-count rate (DCR) respectively. Figure $3 \mathrm{~b}$ ) shows a timing histogram with and incident pulsed laser (1310 $\mathrm{nm}$ wavelength). These measurements were taken with an electric detector gate length of $50 \mathrm{~ns}$.

\section{RESULTS}

\subsection{Single Photon Detection Efficiency \& Dark Count Rate}

The SPDE and DCR are subsequently calculated for a range of excess biases, which is the percentage above the SPAD breakdown voltage. Figure 4 shows DCR and SPDE at various temperatures for both the $100 \mu \mathrm{m}$ and $26 \mu \mathrm{m}$ diameter devices. Figure 4a) \& b) shows the DCR for $100 \mu \mathrm{m}$ and $26 \mu \mathrm{m}$ diameter devices respectively in the temperature range of 77 to $175 \mathrm{~K}$. As expected, there is a strong temperature dependence due to the Shockley-Read-Hall generation that likely dominates the dark counts. Scaling the diameter to $26 \mu \mathrm{m}$ has a significant effect on reducing DCR, reaching values below 1 kilocounts/s at low temperature and low excess bias. The lower DCR enables use at higher operation temperatures, which were measurable up to $175 \mathrm{~K}$. Compared to the $100 \mu \mathrm{m}$ diameter device there is a significant improvement for comparable temperatures, which is expected due to the reduction in the absolute number of generation-recombination centers in the
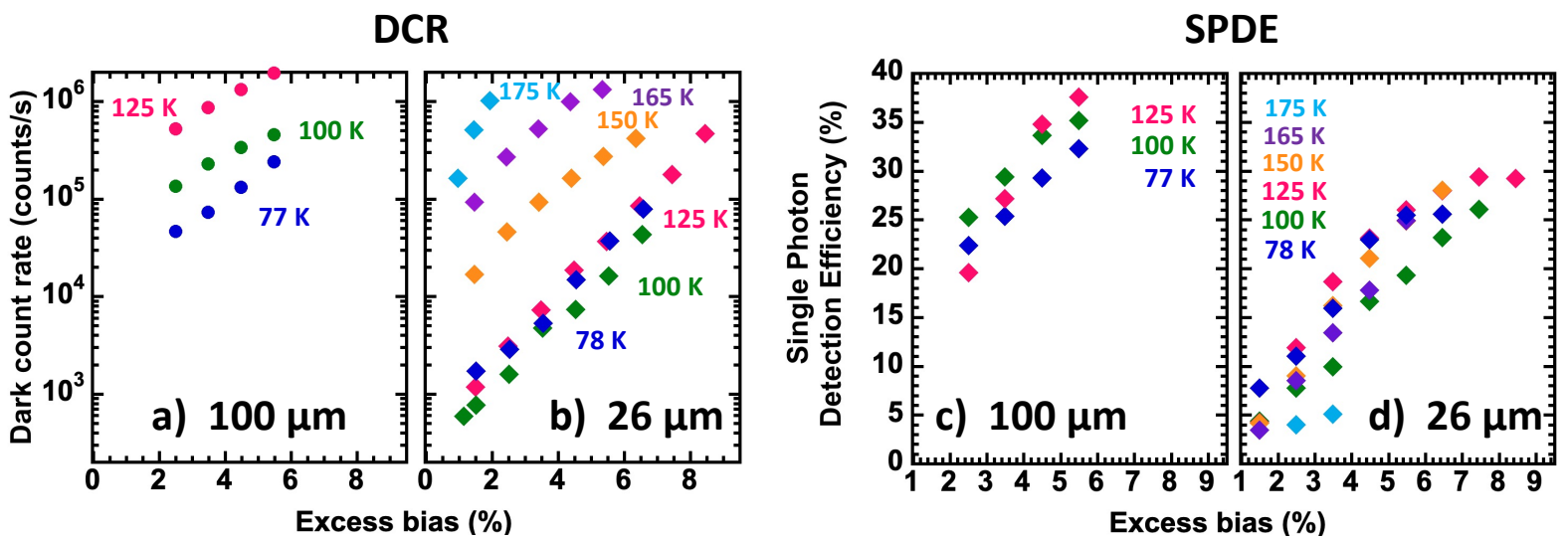

Figure 4: The dark count rate for a) $100 \mu \mathrm{m}$ and b) $26 \mu \mathrm{m}$ diameter pseudo-planar SPADs. c) The single-photon detection efficiency for c) $100 \mu \mathrm{m}$ diameter devices and d) $26 \mu \mathrm{m}$ diameter devices.

smaller pixel. For example, the DCR of a $26 \mu \mathrm{m}$ diameter device at $150 \mathrm{~K}$ is comparable to the DCR of a $100 \mu \mathrm{m}$ diameter device at $77 \mathrm{~K}$. Further scaling the technology is a feasible means to increasing the operation temperature, with the ultimate goal of operating at temperatures compatible with Peltier coolers.

The SPDE, as a function of excess bias, for the $100 \mu \mathrm{m}$ and $26 \mu \mathrm{m}$ diameter devices are shown in Fig. $4 \mathrm{c}) \&$ d) respectively. The $26 \mu \mathrm{m}$ diameter devices reach as high as $29 \%$ at $1310 \mathrm{~nm}$ operated at $\sim 7.5 \%$ excess bias. This is slightly less than the $100 \mu \mathrm{m}$ diameter devices, which reached an SPDE of $38 \%$ at $1310 \mathrm{~nm}$. It is thought that this discrepancy may be due to a spatially non-uniform triggering efficiency across the active area of the $26 \mu \mathrm{m}$ diameter pixel, meaning that the SPDE would appear to reduce when the illuminating laser spot is comparable to the optical active area of the pixel. These results are particularly encouraging, because at these operation temperatures, it is estimated that only approximately $50 \%$ of photons are absorbed in the $1 \mu \mathrm{m}$ thick Ge absorber. In contrast to mesa technology, both devices show substantial improvements compared to $4 \%$ SPDE of a $25 \mu \mathrm{m}$ mesa-etched diameter pixel. The increased SPDE compared to comparable mesa etched technology is likely because a more uniform electric-field in the pseudo-planar structure reduces edge breakdown, meaning that the photogenerated carriers are multiplied efficiently in the active area of the pixel. In 
contrast, in the mesa design, the device breakdown may have been dominated by edge effects, meaning the central region of the device where photogenerated carriers are predominantly collected would be at a lower electric field where multiplication is less efficient. In previous work, the afterpulsing of a pseudo-planar $100 \mu \mathrm{m}$ diameter device was shown to be around $20 \%$ that of a commercial InGaAs/InP device when operated in identical conditions [14]. Afterpulsing has yet to be measured on $26 \mu \mathrm{m}$ devices however reducing the diameter is likely to further reduce afterpulsing which is encouraging for high repetition rate applications.

\subsection{Jitter}

Scaling the SPAD pixel diameters has further benefits beyond reducing the DCR. The jitter, defined as the full-width half maximum of the timing histogram under illumination, is seen to reduce to a minimum of $134 \pm 10 \mathrm{ps}$ at $100 \mathrm{~K}$ compared to the $310 \mathrm{ps}$ measured on previous $100 \mu \mathrm{m}$ diameter devices. The jitter is seen to increase to $204 \pm 10 \mathrm{ps}$ at $165 \mathrm{~K}$. This is an indication of the temporal uncertainty on a measurement, and in turn on distance in a LIDAR system. A lower jitter reduces the required acquisition time in a LIDAR measurement for an equivalent signal-to-noise ratio. As shown in Fig. 5a), the jitter is a strong function of the excess bias, due to the fact that the avalanche build-up variance is a contributing factor. All jitter measurements shown here were taken in an optical cryostat with non-optimized cabling, and it is expected that the jitter will reduce with improved packaging.
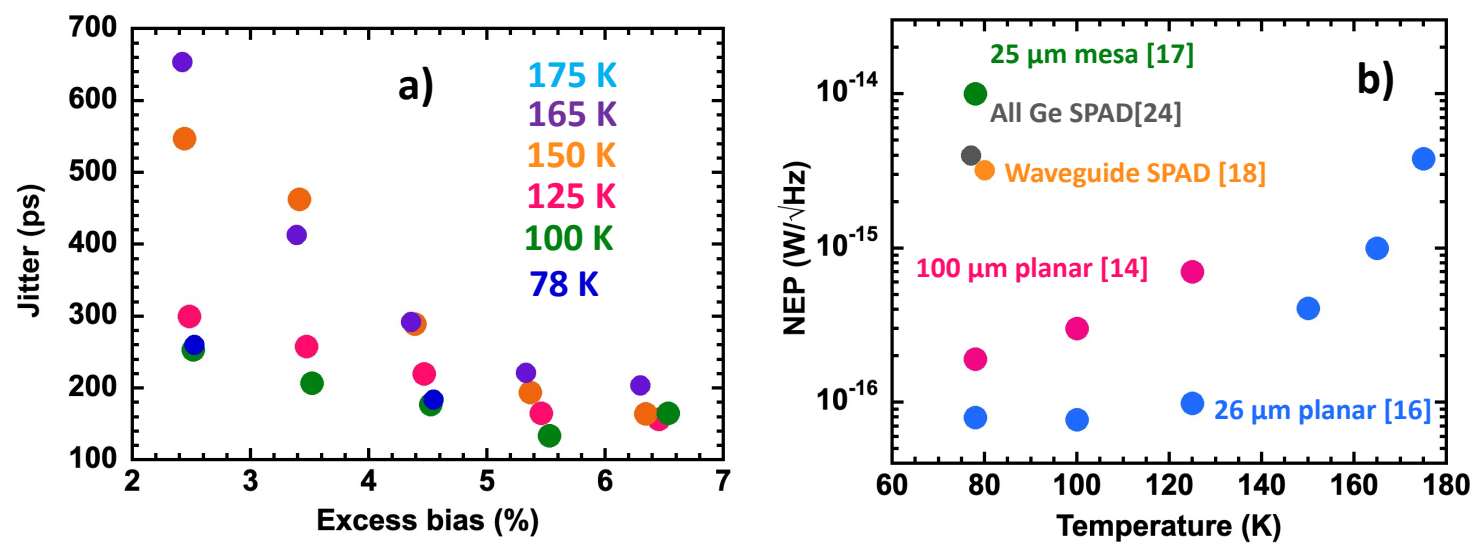

Figure 5: a) The measured jitter of a 26 um diameter pseudo-planar SPAD as a function of excess bias for a range of temperatures. b) The noise-equivalent powers for a range of Ge based SPADs as a function of the temperature.

\subsection{Noise-Equivalent Power}

The improvement in device sensitivity is clearly demonstrated when examining the noise-equivalent-power, which for a SPAD is defined as $N E P=\frac{h v}{\operatorname{SPDE}} \sqrt{2 D C R}$, where $\mathrm{h}$ is Planck's constant and $v$ is the frequency of operation. The NEP for a range of devices measured at $1310 \mathrm{~nm}$ wavelength [14,16-18,24] are demonstrated in Fig. 5b). Comparing the pseudoplanar $26 \mu \mathrm{m}$ diameter device to a comparable $25 \mu \mathrm{m}$ mesa diameter device demonstrates there is over a 2 order of magnitude reduction in NEP, reaching as low as $7.7 \times 10^{-17} \mathrm{WHz}^{-1 / 2}$ at $100 \mathrm{~K}$. The pseudo-planar technology demonstrates significantly lower NEP than waveguide geometry devices [18] despite having a Ge absorber area increase of $\sim 30$ times. This is testament to the fact that the pseudo-planar design has caused a step-change in performance and highlights that all previous mesa demonstrations likely suffered from a high sidewall interaction and from electric field non-uniformities that are highly detrimental to SPAD performance. These results, however, need to be compared to the state-of-the-art InGaAs/InP SPADs which demonstrate NEPs of $5 \times 10^{-17} \mathrm{WHz}^{-1 / 2}$ at $230 \mathrm{~K}$ with $1550 \mathrm{~nm}$ wavelength operation [Micro Photon Devices Srl. PDM-IR $25 \mu \mathrm{m}$ diameter InGaAs/InP SPAD datasheet] indicating that further optimization is required to achieve comparable performance to the best III-V SPADs in the SWIR.

\subsection{Light Detecton and Ranging performance}

The performance of the $26 \mu \mathrm{m}$ diameter pixels for LIDAR and ranging was evaluated using the LIDAR equation. The full assumptions for the model are described in [25], but it assumes conservative estimates of optical system losses of $10 \mathrm{~dB}$, and $10 \%$ reflectivity from a Lambertian reflector (i.e. the target). Figure 6 shows the required laser power for ranging as a function of distance, for a variety of integration times. This was modelled using the measured SPDEs of $15 \%$ and $9 \%$ for 
$1310 \mathrm{~nm}$ and $1450 \mathrm{~nm}$ wavelength respectively, using an excess bias of $2.5 \%$ and operated at $125 \mathrm{~K}$. The DCR for this device was 2.6 kilocounts/s. The results demonstrate that with the measured device metrics (SPDE, DCR, jitter), kilometer
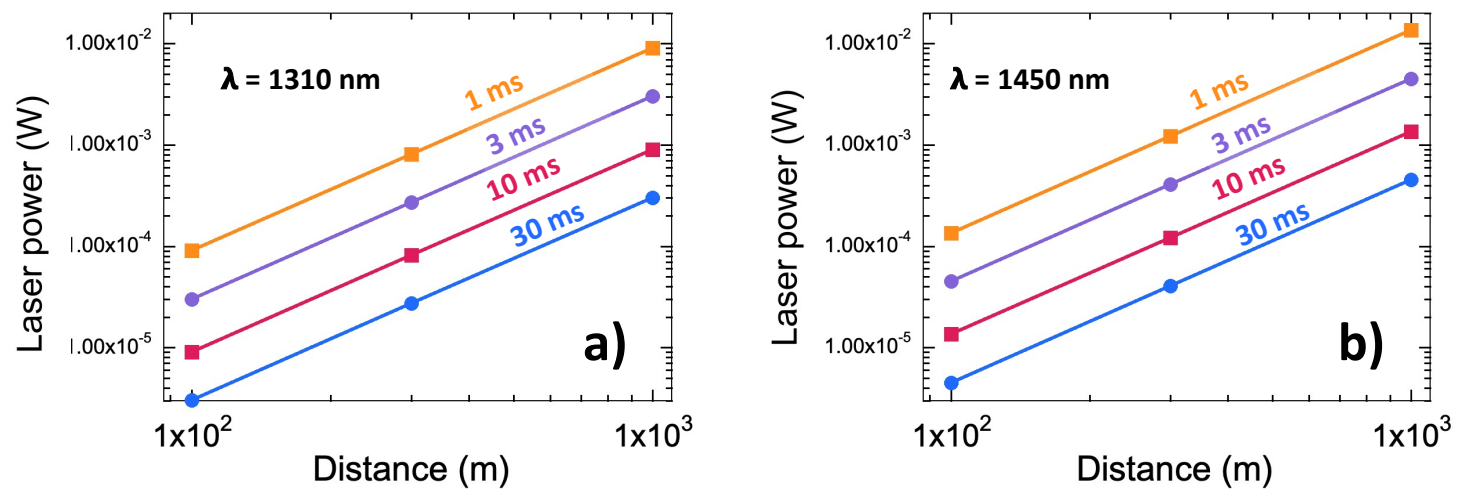

Figure 6: The average laser power required to image a target as a function of the object distance for a range of integration times, using a $26 \mu \mathrm{m}$ diameter SPAD operated at a) $1310 \mathrm{~nm}$ and b) $1450 \mathrm{~nm}$.

range-finding is achievable with eye-safe laser powers, in the absence of atmospheric obscurants. It can be observed that per pixel acquisition times as low as $1 \mathrm{~ms}$ are feasible at $1310 \mathrm{~nm}$ wavelength, with an object distance of $1 \mathrm{~km}$, while remaining eye safe. The averaging time at $1450 \mathrm{~nm}$ wavelength increases slightly for a given distance in this case due to the reduction of SPDE at $1450 \mathrm{~nm}$, due to the SPAD operating close to the direct band-gap band-edge of Ge. For imaging applications, there is potential to reduce the total acquisition time by using partial information about the target, and subsequently reconstructing the profiles using algorithms such as the Restoration of Depth and Intensity using Total Variation (RDI-TV) algorithm and the Manifold Point Process (ManiPoP) algorithm [26,27]. On a per-pixel basis, there are further means of reducing acquisition times further is by enhancing the absorption and extension to longer wavelengths, which is discussed in the following section.

\section{FUTURE WORK}

The pseudo-planar results to date have shown SPDEs at wavelengths $<1550 \mathrm{~nm}$. Further work is required to extend the operation wavelength further into the SWIR. In particular, for eye-safe LIDAR, operation at wavelengths beyond $1400 \mathrm{~nm}$ is important so as to extend beyond the retinal hazard window, enabling higher laser powers and ultimately allowing for longer range measurements. For military range-finding, wavelengths of $1535 \mathrm{~nm}$ are often used for long-range measurements due to a combination of eye-safety and atmospheric transmission [28]. For many quantum applications, operation at $1550 \mathrm{~nm}$ is a key target wavelength for compatibility with telecoms systems. Currently, one limiting factor is the operation temperature, and the temperature dependence of the Ge bandgap. Optimizing the technology to reduce DCR (e.g. with scaling) will enable higher temperature operation and correspondingly the absorption will red-shift to longer wavelengths. Absorption can further be increased by increasing the thickness of the avalanche layer, with absorber thicknesses of approximately 2-3 $\mu \mathrm{m}$ being feasible with current epitaxial growth techniques [29]. Modelling demonstrates that operation at $\sim 240 \mathrm{~K}$ with a $2 \mu \mathrm{m}$ thick absorber would enable absorption of $>25 \%$ of photons at $1550 \mathrm{~nm}$. This could further be improved by moving to back-side illuminated wafers, with a top-side reflector such that incoming photons go through an optical double pass of the absorber layer. This has the advantage of doubling the optical pathlength while maintaining the same device volume to keep DCR to a minimum.

A longer-term goal is to include small fractions of Sn in the absorber layer; Sn alloying modifies the Ge bandstructure and red-shifts absorption [30], however a low temperature growth is required to enable Sn incorporation [31], which can lead to additional defect states that could be detrimental to DCR. Tensile strain is another means by which absorption can be extended, having a similar effect to Sn alloying. High levels of strain by $\mathrm{Si}_{\mathrm{x}} \mathrm{N}_{\mathrm{y}}$ stressor layers are transferable to nanofeatures or undercut cavities [32-34], however this approach is not readably applicable to feature sizes more than several microns. This technique may however be applicable to waveguide geometry devices for quantum applications on SOI material. 


\section{CONCLUSION}

A record low NEP of $7.7 \times 10^{-17} \mathrm{WHz}^{-1 / 2}$ has been demonstrated with pseudo-planar Ge-on-Si SPADs with $26 \mu \mathrm{m}$ diameter charge sheet regions. This has resulted in a step-change in performance from $100 \mu \mathrm{m}$ diameter pixels, and has shown a 2 order-of-magnitude reduction in NEP compared to $25 \mu \mathrm{m}$ diameter mesa devices reported previously. Scaling the pixel technology has enabled single-photon counting up to $175 \mathrm{~K}$, and has reduced the temporal jitter down to $134 \pm$ $10 \mathrm{ps}$. Single photon detection efficiencies were found to reach up to $29 \%$ with $1 \mu \mathrm{m}$ thick absorbers, showing there is scope to improve this significantly with thicker absorber layers and by further scaling the technology to reduce DCR. These developments show that there is real promise for Ge-on-Si technology as a low-cost, low afterpulsing, alternative to InGaAs/InP for SPADS operating in the SWIR, for imaging and quantum applications.

\section{REFERENCES}

[1] Buller, G. S. and Collins, R. J., "Single-photon generation and detection," Meas. Sci. Technol. 21(1), 012002 (2010).

[2] Bird, R. E., Hulstrom, R. L. and Lewis, L. J., “Terrestrial solar spectral data sets,” Sol. Energy 30(6), 563-573 (1983).

[3] Tobin, R., Halimi, A., McCarthy, A., Laurenzis, M., Christnacher, F. and Buller, G. S., "Three-dimensional single-photon imaging through obscurants," Opt. Express 27(4), 4590 (2019).

[4] McCarthy, A., Ren, X., Della Frera, A., Gemmell, N. R., Krichel, N. J., Scarcella, C., Ruggeri, A., Tosi, A. and Buller, G. S., "Kilometer-range depth imaging at $1550 \mathrm{~nm}$ wavelength using an InGaAs/InP single-photon avalanche diode detector," Opt. Express 21(19), 22098 (2013).

[5] Tobin, R., Halimi, A., McCarthy, A., Soan, P. J. and Buller, G. S., "Robust real-time 3D imaging of moving scenes through atmospheric obscurant using single-photon LiDAR," Sci. Rep. 11(1), 1-13 (2021).

[6] Hadfield, R. H., "Single-photon detectors for optical quantum information applications," Nat. Photonics 3(12), 696-705 (2009).

[7] Diamanti, E., Lo, H.-K., Qi, B. and Yuan, Z., "Practical challenges in quantum key distribution," npj Quantum Inf. 2(1), 16025 (2016).

[8] Collins, R. J., Amiri, R., Fujiwara, M., Honjo, T., Shimizu, K., Tamaki, K., Takeoka, M., Andersson, E., Buller, G. S. and Sasaki, M., "Experimental transmission of quantum digital signatures over $90 \mathrm{~km}$ of installed optical fiber using a differential phase shift quantum key distribution system," Opt. Lett. 41(21), 4883 (2016).

[9] Ziarkash, A. W., Joshi, S. K., Stipčević, M. and Ursin, R., "Comparative study of afterpulsing behavior and models in single photon counting avalanche photo diode detectors," Sci. Rep. 8(1), 5076 (2018).

[10] Loudon, A. Y., Hiskett, P. A., Buller, G. S., Carline, R. T., Herbert, D. C., Leong, W. Y. and Rarity, J. G., "Enhancement of the infrared detection efficiency of silicon photon-counting avalanche photodiodes by use of silicon germanium absorbing layers," Opt. Lett. 27(4), 219 (2002).

[11] Lu, Z., Kang, Y., Hu, C., Zhou, Q., Liu, H.-D. and Campbell, J. C., "Geiger-Mode Operation of Ge-on-Si Avalanche Photodiodes," IEEE J. Quantum Electron. 47(5), 731-735 (2011).

[12] Carroll, M. S., Childs, K., Jarecki, R., Bauer, T. and Saiz, K., "Ge-Si separate absorption and multiplication avalanche photodiode for Geiger mode single photon detection," Appl. Phys. Lett. 93(18), 183511 (2008).

[13] Ke, S., Lin, S., Mao, D., Ye, Y., Ji, X., Huang, W., Li, C. and Chen, S., "Design of wafer-bonded structures for near room temperature Geiger-mode operation of germanium on silicon single-photon avalanche photodiode," Appl. Opt. 56(16), 4646 (2017).

[14] Vines, P., Kuzmenko, K., Kirdoda, J., Dumas, D. C. S., Mirza, M. M., Millar, R. W., Paul, D. J. and Buller, G. S., "High performance planar germanium-on-silicon single-photon avalanche diode detectors," Nat. Commun. 10(1) (2019).

[15] Thorburn, F. E., Huddleston, L. L., Kirdoda, J., Millar, R. W., Ferre Llin, L., Yi, X., Paul, D. J. and Buller, G. S., "High efficiency planar geometry germanium-on-silicon single-photon avalanche diode detectors," Adv. Phot. Count. Tech. XIV(May 2020), M. A. Itzler, K. A. McIntosh, and J. C. Bienfang, Eds., 25, SPIE (2020).

[16] Llin, L. F., Kirdoda, J., Thorburn, F., Huddleston, L. L., Greener, Z. M., Kuzmenko, K., Vines, P., Dumas, D. C. S., Millar, R. W., Buller, G. S. and Paul, D. J., "High sensitivity Ge-on-Si single-photon avalanche diode detectors," Opt. Lett. 45(23), 6406 (2020). 
[17] Warburton, R. E., Intermite, G., Myronov, M., Allred, P., Leadley, D. R., Gallacher, K., Paul, D. J., Pilgrim, N. J., Lever, L. J. M., Ikonic, Z., Kelsall, R. W., Huante-Ceron, E., Knights, A. P. and Buller, G. S., "Ge-on-si single-photon avalanche diode detectors: Design, modeling, fabrication, and characterization at wavelengths 1310 and 1550 nm," IEEE Trans. Electron Devices 60(11), 3807-3813 (2013).

[18] Martinez, N. J. D., Gehl, M., Derose, C. T., Starbuck, A. L., Pomerene, A. T., Lentine, A. L., Trotter, D. C. and Davids, P. S., "Single photon detection in a waveguide-coupled Ge-on-Si lateral avalanche photodiode," Opt. Express 25(14), 16130 (2017).

[19] Colace, L., Masini, G., Galluzzi, F., Assanto, G., Capellini, G., Di Gaspare, L., Palange, E. and Evangelisti, F., "Metal-semiconductor-metal near-infrared light detector based on epitaxial Ge/Si," Appl. Phys. Lett. 72(24), 3175-3177 (1998).

[20] Mirza, M. M., Zhou, H., Velha, P., Li, X., Docherty, K. E., Samarelli, A., Ternent, G. and Paul, D. J., "Nanofabrication of high aspect ratio ( 50:1) sub-10 nm silicon nanowires using inductively coupled plasma etching,” J. Vac. Sci. Technol. B, Nanotechnol. Microelectron. Mater. Process. Meas. Phenom. 30(6), 06FF02 (2012).

[21] Mirza, M. M., MacLaren, D. A., Samarelli, A., Holmes, B. M., Zhou, H., Thoms, S., MacIntyre, D. and Paul, D. J., "Determining the Electronic Performance Limitations in Top-Down-Fabricated Si Nanowires with Mean Widths Down to 4 nm," Nano Lett. 14(11), 6056-6060 (2014).

[22] Xie, M., Nishimura, T., Yajima, T. and Toriumi, A., "Reaction of $\mathrm{GeO}_{2}$ with $\mathrm{Ge}$ and crystallization of $\mathrm{GeO}_{2}$ on Ge,” J. Appl. Phys. 127(2), 2-7 (2020).

[23] Pezzoli, F., Giorgioni, A., Gallacher, K., Isa, F., Biagioni, P., Millar, R. W., Gatti, E., Grilli, E., Bonera, E., Isella, G., Paul, D. J. and Miglio, L., "Disentangling nonradiative recombination processes in Ge micro-crystals on Si substrates," Appl. Phys. Lett. 108(26) (2016).

[24] Tosi, A., Dalla Mora, A., Zappa, F. and Cova, S., "Germanium and InGaAs/InP SPADs for single-photon detection in the near-infrared," Adv. Phot. Count. Tech. II 6771(October 2007), W. Becker, Ed., 67710P (2007).

[25] Kuzmenko, K., Vines, P., Halimi, A., Collins, R. J., Maccarone, A., McCarthy, A., Greener, Z. M., Kirdoda, J., Dumas, D. C. S., Llin, L. F., Mirza, M. M., Millar, R. W., Paul, D. J. and Buller, G. S., "3D LIDAR imaging using Ge-on-Si single-photon avalanche diode detectors," Opt. Express 28(2), 1330 (2020).

[26] Halimi, A., Altmann, Y., McCarthy, A., Ren, X., Tobin, R., Buller, G. S. and McLaughlin, S., "Restoration of intensity and depth images constructed using sparse single-photon data," Eur. Signal Process. Conf. 2016Novem, 86-90, IEEE (2016).

[27] Tachella, J., Altmann, Y., Ren, X., McCarthy, A., Buller, G. S., McLaughlin, S. and Tourneret, J.-Y., "Bayesian 3D Reconstruction of Complex Scenes from Single-Photon Lidar Data," SIAM J. Imaging Sci. 12(1), 521-550 (2019).

[28] Maini, A. K., [Handbook of Defence Electronics and Optronics], John Wiley \& Sons, Ltd, Chichester, UK (2018).

[29] Gallacher, K., Millar, R. W., Griškevičiūte, U., Baldassarre, L., Sorel, M., Ortolani, M. and Paul, D. J., "Low loss Ge-on-Si waveguides operating in the 8-14 $\mu \mathrm{m}$ atmospheric transmission window," Opt. Express 26(20), 25667 (2018).

[30] Millar, R. W., Dumas, D. C. S., Gallacher, K. F., Jahandar, P., MacGregor, C., Myronov, M. and Paul, D. J., "Mid-infrared light emission $>3 \mu \mathrm{m}$ wavelength from tensile strained GeSn microdisks," Opt. Express 25(21), 25374 (2017).

[31] Wirths, S., Buca, D. and Mantl, S., "Si-Ge-Sn alloys: From growth to applications," Prog. Cryst. Growth Charact. Mater. 62(1), 1-39 (2016).

[32] Bashir, A., Millar, R. W., Gallacher, K., Paul, D. J., Darbal, A. D., Stroud, R., Ballabio, A., Frigerio, J., Isella, G. and MacLaren, I., "Strain analysis of a Ge micro disk using precession electron diffraction," J. Appl. Phys. 126(23) (2019).

[33] Millar, R. W., Gallacher, K., Frigerio, J., Ballabio, A., Bashir, A., MacLaren, I., Isella, G. and Paul, D. J., "Analysis of Ge micro-cavities with in-plane tensile strains above 2 \%," Opt. Express 24(5), 4365 (2016).

[34] Ghrib, A., El Kurdi, M., Prost, M., de Kersauson, M., Largeau, L., Mauguin, O., Beaudoin, G., Sauvage, S., Checoury, X., Ndong, G., Chaigneau, M., Ossikovski, R., David, S., Sagnes, I. and Boucaud, P., "Strain engineering in germanium microdisks," Silicon Photonics IX 8990, 89901C (2014). 